\title{
Statine per orbitopatia di Graves (STAGO): un trial clinico di fase 2, randomizzato, open-label e monocentrico
}

\author{
Arianna Cremaschi ${ }^{1}$. Giovanna Mantovani ${ }^{1}$
}

Accettato: 30 dicembre 2021 / Pubblicato online: 3 marzo 2022

c) The Author(s) 2022, corrected publication 2022

Commento a:

Statins for Graves' orbitopathy (STAGO): a phase 2, open-label, adaptive, single centre, randomised clinical trial.

G. Lanzolla, E. Sabini, M. Leo, F. Menconi, R. Rocchi, A. Sframeli, P. Piaggi, M. Nardi, C. Marcocci, M. Marinò. Lancet Diabetes Endocrinol (2021) 9:733-742

Diversi lavori hanno dimostrato una correlazione fra colesterolo LDL e orbitopatia di Graves (GO) e due studi trasversali hanno dimostrato un'azione protettiva delle statine sullo sviluppo di GO [1, 2]; tali dati suggeriscono, pertanto, un potenziale uso di questi farmaci anche nel trattamento della patologia. Lo scopo del presente studio era di valutare l'efficacia dell' aggiunta di una statina, in particolare dell'atorvastatina in quanto il più utilizzato della sua classe, a uno schema infusionale di glucocorticoidi (GC) nell'outcome di pazienti con GO e ipercolesterolemia [3].

Trattasi di un clinical trial di fase 2 randomizzato, openlabel e adattativo in un singolo centro di terzo livello all'Ospedale Universitario di Pisa. I criteri di inclusione erano la presenza di GO attiva di grado moderato-severo e di ipercolesterolemia, definita da valori di colesterolo LDL tra 2,97 e $4,88 \mathrm{mmol} / \mathrm{L}$. I pazienti sono stati randomizzati (1:1) in due gruppi: un gruppo ricevente metilprednisolone e atorvastatina al dosaggio di $20 \mathrm{mg}$ die per 24 settimane (gruppo ST) e un gruppo ricevente metilprednisolone (gruppo NST). Lo schema infusionale di glucocorticoidi era il medesimo per entrambi i gruppi e prevedeva metilprednisolone al dosaggio di $500 \mathrm{mg}$, un'infusione a settimana per 6 settimane e a seguire $250 \mathrm{mg}$ un'infusione a settimana per ulteriori 6 settimane. Pazienti e medici del team erano resi edotti del trat-

G. Mantovani

giovanna.mantovani@unimi.it

1 Unità di Endocrinologia, Fondazione IRCCS Ca' Granda Ospedale Maggiore Policlinico, Dipartimento di Scienze Cliniche e di Comunità, Università degli Studi di Milano, Milano, Italia tamento ricevuto, ad esclusione dei colleghi oftalmologi che risultavano in cieco. L'endpoint primario era l'outcome della GO, esaminato a 24 settimane di trattamento, basandosi su una valutazione composita. In particolare, venivano considerati responders i pazienti in cui almeno due dei seguenti criteri venivano realizzati: 1) riduzione di almeno $2 \mathrm{~mm}$ dell'esoftalmo senza incremento superiore ai $2 \mathrm{~mm}$ nell'occhio controlaterale; 2) riduzione del CAS (clinical activity score) di almeno 2 punti; 3) riduzione della retrazione palpebrale di almeno $2 \mathrm{~mm}$, senza incrementi di oltre $2 \mathrm{~mm}$ nell'occhio controlaterale; 4) scomparsa o miglioramento della diplopia; 5) miglioramento dell'acuità visiva di almeno 0,2 decimi. Gli outcome secondari valutati erano l'outcome dell'orbitopatia a 12 settimane di trattamento, modifiche in qualità della vita (QoL) e recidiva di GO a 24 settimane, definita come un peggioramento di almeno 2 degli stessi 5 criteri precedentemente elencati rispetto al controllo della 12 settimana.

Complessivamente, la popolazione includeva 41 pazienti nel gruppo ST e 39 nel gruppo NST. Nel gruppo ST si assisteva a una riduzione significativa dei valori di LDL rispetto al gruppo NST (differenza media di $-0,4 \mathrm{mmol} / \mathrm{L} ; 95 \% \mathrm{CI}$ da $-0,7$ a - 0,1$)$. Usando la valutazione composita, la proporzione di pazienti con GO responders a 24 settimane era significativamente più elevata nel gruppo ST rispetto al gruppo NST (51 vs 28\%; AR 0,23; $p=0,015$ ). Per quanto riguarda gli outcome secondari si è evidenziata una differenza statisticamente significativa in termini di modifiche di QoL fra il gruppo ST e il gruppo NST a 24 settimane (differenza media 6,4 punti; 95\% CI 0,6-12,1; $p=0,031$ ); nessuno nel gruppo ST ha avuto una recidiva di GO, mentre 6 dei 39 pazienti del gruppo NST (15\%) hanno avuto una recidiva; vi è stato un miglioramento significativo in termini di CAS (differenza media -1 punto; 95\% CI da -1 a $-1 ; p<0,0001$ ). Non vi è stata, invece, una differenza significativa nelle modifiche dei singoli caratteri oculari analizzati, così come nei valori di FT4 e di TRAb. È stato studiato l'effetto di sesso, età, abitudine tabagica, terapia radiometabolica, TRAb, durata di malattia, trattamento con prednisone, bassa concentrazione 
di LDL di base e utilizzo di atorvastatina e l'unica variabile con impatto significativo sull'outcome della GO a 24 settimane era l'uso di atorvastatina. Per determinare se l'effetto dell' atorvastatina fosse legato alla capacità ipolipemizzante, sono stati confrontati i valori ematici di LDL tra i pazienti responders e non responders nel gruppo ST; tuttavia, non si sono evidenziate differenze significative.

L'effetto delle statine potrebbe essere correlato al meccanismo ipolipemizzante. È noto, infatti, che l'ipercolesterolemia ha azioni proinfiammatorie sistemiche tramite meccanismi di stress ossidativo, rilascio di citochine proinfiamamtorie, alcune delle quali hanno un ruolo nella patogenesi della GO e overespressione del gene TLR4 nei fibroblasti orbitali. Tuttavia, altri meccanismi pleiotropici potrebbero essere coinvolti, tra cui effetti sulla regolazione della tolleranza immunitaria, sull'infiammazione o sul rimodellamento tissutale. Infine, vi potrebbero essere delle interazioni farmacologiche con il metilprednisolone. L'atorvastatina è metabolizzata e risulta essere un inibitore di CYP3A4; pertanto, la terapia combinata potrebbe incrementare gli effetti dei glucocorticoidi.

In conclusione, l'aggiunta di atorvastatina allo schema di glucocorticoidi endovena migliora la risposta della GO associata a ipercolesterolemia. Inoltre, non sono state osservate differenze nel numero o nella gravità degli eventi avversi fra i due gruppi, suggerendo che l'atorvastatina non incrementa i rischi legati al trattamento.

Funding Note Open access funding provided by Università degli Studi di Milano within the CRUI-CARE Agreement.
Open Access This article is licensed under a Creative Commons Attribution 4.0 International License, which permits use, sharing, adaptation, distribution and reproduction in any medium or format, as long as you give appropriate credit to the original author(s) and the source, provide a link to the Creative Commons licence, and indicate if changes were made. The images or other third party material in this article are included in the article's Creative Commons licence, unless indicated otherwise in a credit line to the material. If material is not included in the article's Creative Commons licence and your intended use is not permitted by statutory regulation or exceeds the permitted use, you will need to obtain permission directly from the copyright holder. To view a copy of this licence, visit http://creativecommons.org/licenses/by/4. $0 \%$.

\section{Bibliografia}

1. Stein JD, Childers D, Gupta S et al (2015) Risk factors for developing thyroid-associated ophthalmopathy among individuals with Graves disease. JAMA Ophthalmol 133:290-296

2. Nilsson A, Tsoumani K, Planck T (2021) Statins decrease the risk of orbitopathy in newly diagnosed patients with Graves disease. J Clin Endocrinol Metab 106:1325-1332

3. Lanzolla G, Sabini E, Leo M et al (2021) Statins for Graves' orbitopathy (STAGO): a phase 2, open-label, adaptive, single centre, randomised clinical trial. Lancet Diabetes Endocrinol 9:733-742

Nota della casa editrice Springer Nature rimane neutrale in riguardo alle rivendicazioni giurisdizionali nelle mappe pubblicate e nelle affiliazioni istituzionali. 